\title{
Pyoderma gangrenosum associated with chronic refractory pouchitis: a case successfully treated with infliximab
}

\author{
Dimitra Koumaki ${ }^{a}$, Eleni Orfanoudaki ${ }^{b}$, Angeliki Machairab ${ }^{b}$ Eleni Lagoudakic, Konstantinos Krasagakis ${ }^{a}$, \\ loannis E. Koutroubakis ${ }^{b}$
}

University Hospital of Heraklion, Crete, Greece

\begin{abstract}
Pouchitis-associated pyoderma gangrenosum (PG) is rare, with only a few cases reported in the literature. Here we report a rare case of chronic refractory pouchitis-associated PG successfully treated with infliximab (IFX). A 43-year-old Caucasian male, with a past medical history of chronic refractory pouchitis after proctocolectomy and ileal pouch-anal anastomosis for severe ulcerative colitis, developed PG on his right lower leg. This subsided after treatment with intravenous IFX at a dose of $5 \mathrm{mg} / \mathrm{kg}$ at weeks $0,2,6$ and then every 8 weeks. Pouchitis-associated PG is rare. Clinicians should be aware of the risk of PG in patients who suffer from pouchitis and develop rapidly extensive painful ulcers. Furthermore, the therapeutic choice should take into consideration the effectiveness of IFX on the inflammatory background, which sustains both intestinal and skin disease in these types of patients.
\end{abstract}

Keywords Pouchitis, ulcerative colitis, inflammatory bowel disease, pyoderma gangrenosum, infliximab

Ann Gastroenterol 2020; 33 (4): 1-3

\section{Introduction}

Pyodermagangrenosum (PG) is a rareneutrophilic dermatosis that presents as an inflammatory and ulcerative disorder of the skin [1]. More than half of patients with PG develop the disorder in association with an underlying systemic disease [1]. Inflammatory bowel disease (IBD), hematologic disorders, and arthritis represent the most frequent comorbidities [1]. Pouchitis is an inflammatory condition of the ileal pouch reservoir of an ileal pouch-anal anastomosis (IPAA) [2] after surgery for ulcerative colitis (UC) [2]. Extraintestinal manifestations, including PG, are often associated with UC, but the vast majority resolve after surgery. There are only a few reports in the literature on pouchitis-associated PG [3-6]. Here we report a rare case of a patient with pouchitis-associated PG successfully treated with infliximab (IFX).

Department of a Dermatology (Dimitra Koumaki, Konstantinos Krasagakis); 'Gastroenterology (Eleni Orfanoudaki, Angeliki Machaira, Ioannis E. Koutroubakis); 'Pathology (Eleni Lagoudaki), University Hospital of Heraklion, Crete, Greece

Conflict of Interest: None

Correspondence to: Dimitra Koumaki, Dermatology Department, University Hospital of Heraklion, Voutes, Heraklion, 71110 Crete, Greece, e-mail dkoumaki@yahoo.gr

Received 23 April 2020; accepted 29 April 2020; published online 28 May 2020

DOI: https://doi.org/10.20524/aog.2020.0500

\section{Case report}

In January 2020, a 43-year-old Caucasian male attended the accident and emergency department of our hospital for a painful deep purulent ulceration with a violaceous border on the right lower leg that had developed rapidly after trauma in the previous 4 months. His past medical history was significant for severe extensive UC since the age of 19. Restorative proctocolectomy with IPAA (J-pouch) became necessary 6 years after diagnosis, because of severe chronic activity and disease refractory to oral and intravenous corticosteroids and oral mesalamine. Biopsies of the surgical specimen were compatible with UC; no signs of Crohn's disease or indeterminate colitis were found. Since then he had developed severely active chronic pouchitis intermittently treated with oral ciprofloxacin, metronidazole, budesonide and probiotics (VSL\#3). He was an ex-smoker and reported $8-10$ bowel movements a day.

On clinical examination, there was a $6 \times 4 \mathrm{~cm}$ painful ulcer with purulent and necrotic base and violaceous borders in a serpiginous configuration on the right lower leg (Fig. 1). Clinically, the differential diagnosis included skin and soft tissue infection (SSTI), PG, cutaneous squamous cell carcinoma, deep fungal and atypical mycobacterial infection. C-reactive protein was elevated $(3.34 \mathrm{mg} / \mathrm{dL}$, normal levels $<0.8 \mathrm{mg} / \mathrm{dL}$ ). Other biomarkers were normal, including hemoglobin $13.7 \mathrm{~g} / \mathrm{dL}$, white blood cell count $7.8 \mathrm{~K} / \mu \mathrm{L}$, platelet count $389 \mathrm{~K} / \mu \mathrm{L}$, erythrocyte sedimentation rate $13 \mathrm{~mm} / \mathrm{h}$. Serology for antinuclear antibodies, extractable nuclear antigen, anti-ds DNA, and rheumatoid factor was also negative. 


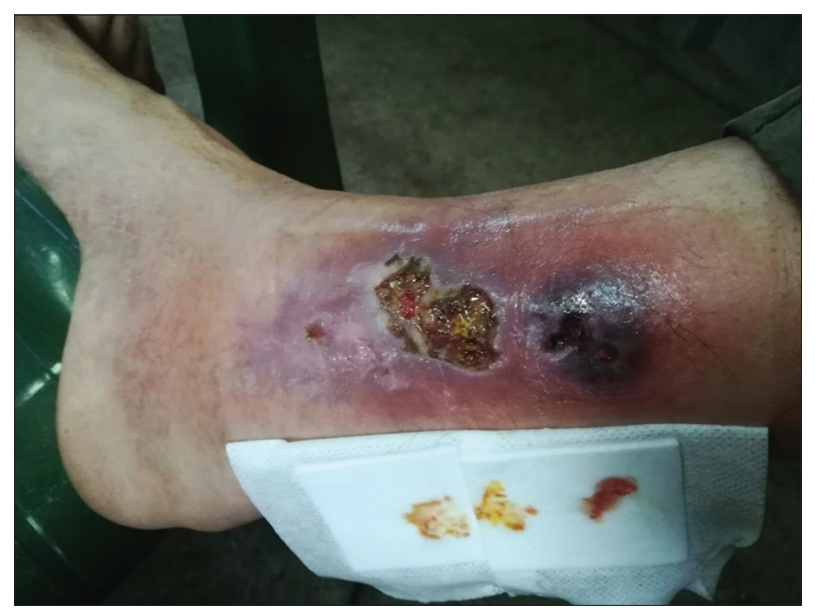

Figure 1 Ulcer with purulent and necrotic base and violaceous borders on the right lower leg

The patient was admitted to the hospital and was initially treated as having SSTI of the right lower leg. Pseudomonas aeruginosa was isolated from the wound swab and was found sensitive to piperacillin/tazobactam, ceftazidime, meropenem, and amikacin, but resistant to pefloxacin, minocycline and trimethoprim, and sulfonamides. Tissue cultures were negative for aerobic and anaerobic bacteria, fungal infection and mycobacteria. Blood and stool cultures, Clostridium difficile toxins $\mathrm{A}$ and $\mathrm{B}$, Mantoux tuberculin skin test and QuantiFERON-TB Gold were all negative. He was given intravenous (i.v.) piperacillin/tazobactam $4.5 \mathrm{~g}$ t.i.d. and i.v. daptomycin $4 \mathrm{mg} / \mathrm{kg}$ q.d. for 14 days. A magnetic resonance imaging (MRI) scan of the right lower leg showed diffuse edema of the cellular adipose tissue of the gastrocnemius muscle with contrast enhancement of the affected soft tissue, forming subcutaneous fluid collections indicative of an inflammatory process of the soft tissues of the gastrocnemius muscle. Histology from a skin biopsy from the edge of the ulcer of the right lower leg demonstrated a neutrophilic infiltrate in keeping with PG (Fig. 2). Endoscopic evaluation of the ileal pouch (pouchoscopy) showed inflammation, erythema and multiple ulcers of the ileal pouch with stenosis of the afferent loop (Fig. 3). Pouch biopsies showed small bowel mucosa with crypt architectural distortion and chronic inflammatory infiltration by neutrophils, eosinophils, lymphocytes and plasma cells-features of chronic pouchitis. No pelvic MRI was performed. The pouchitis disease activity index was 13 . The patient was started on treatment with IFX i.v. $5 \mathrm{mg} / \mathrm{kg}$ at 0 , 2 and 6 weeks and then every 8 weeks. During IFX therapy no additional treatment, including corticosteroids, was administered. There was a great improvement in the PG one week after the initiation of IFX treatment. At his follow up after 7 weeks, the patient's gastrointestinal symptoms had improved, with a significant reduction in the number of bowel movements to 6 per day. The patient remains under long-term follow up with the gastroenterology department.

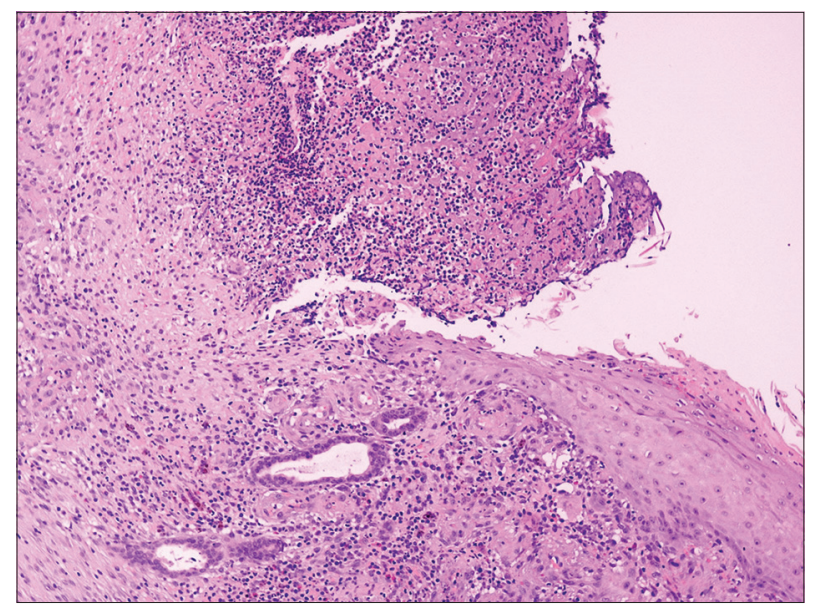

Figure 2 Histology of a skin biopsy of the affected area revealed epidermal and superficial dermal necrosis with an underlying neutrophil infiltrate and lymphocytic vasculitis (hematoxylin and eosin stain, magnification $\times 100$ )

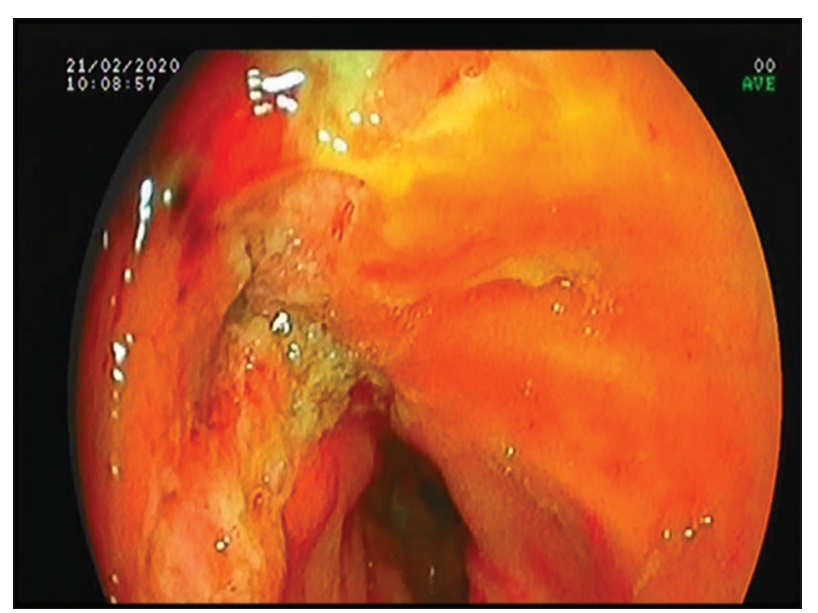

Figure 3 Pouchoscopy view showing mucosal edema, erythema, friability and multiple ulcers

\section{Discussion}

PG is a rare inflammatory neutrophilic skin disorder, whose most common presentation is an inflammatory papule or pustule that progresses to a painful ulcer with a violaceous undermined border and a purulent base, mainly on the lower extremities [1]. Its estimated incidence ranges from 3-10 cases per million people per year [1]. PG most commonly develops in young and middle-aged adults, predominantly in females, with an average age of onset between 40 and 60 years. PG is characterized by neutrophil-predominant infiltrates in the skin [1]. The etiology for the development of the inflammatory process that leads to PG remains unclear; however, proinflammatory cytokines involved in leukocyte function, such as interleukin (IL)-8 and IL-23, seem to play an important role [1]. In addition, the response of PG to IFX and other antitumor necrosis factor (TNF)- $\alpha$ agents suggests an important role for TNF- $\alpha$ in PG [1]. 
Table 1 Summary of demographics, clinical characteristics and treatment of previously reported pouchitis-associated pyoderma gangrenosum

\begin{tabular}{|c|c|c|c|c|c|c|c|c|c|c|c|c|}
\hline Author & City & Country & Year & Sex & $\begin{array}{l}\text { Age of } \\
\text { patient }\end{array}$ & $\begin{array}{c}\text { Age of } \\
\text { UC onset }\end{array}$ & $\begin{array}{c}\text { UC } \\
\text { duration }\end{array}$ & $\begin{array}{l}\text { Age at } \\
\text { surgery }\end{array}$ & $\begin{array}{l}\text { Age at } \\
\text { PG }\end{array}$ & $\begin{array}{l}\text { Type of } \\
\text { pouchitis }\end{array}$ & $\begin{array}{l}\text { Location } \\
\text { of PG }\end{array}$ & $\begin{array}{l}\text { Treatment for } \\
\text { PG }\end{array}$ \\
\hline $\begin{array}{l}\text { Abdelrazeq } \\
\text { et al [3] }\end{array}$ & York & UK & 2004 & $\mathrm{~F}$ & 43 & 16 & 27 & 29 & 37 & Acute & Leg & $\begin{array}{l}\text { Oral } \\
\text { metronidazole } \\
400 \mathrm{mg} \text { tds }\end{array}$ \\
\hline $\begin{array}{l}\text { Yanaru- } \\
\text { Fujisawa [4] }\end{array}$ & Fukuoka & Japan & 2005 & $\mathrm{~F}$ & 31 & 18 & 13 & 20 & 31 & $\begin{array}{l}\text { Chronic } \\
\text { severe }\end{array}$ & $\begin{array}{l}\mathrm{L} \text { ankle } \\
\text { and } \mathrm{R} \text { knee }\end{array}$ & $\begin{array}{l}\text { GCAP } \\
\text { and oral } \\
\text { prednisolone }\end{array}$ \\
\hline $\begin{array}{l}\text { Molnar } \\
\text { et al [5] }\end{array}$ & Szeged & Hungary & 2008 & $\mathrm{~F}$ & 16 & 14 & 2 & 16 & 16 & $\begin{array}{l}\text { Chronic } \\
\text { severe }\end{array}$ & Ileal pouch & Infliximab \\
\hline $\begin{array}{l}\text { Satake } \\
\text { et al [6] }\end{array}$ & Hirosaki & Japan & 2018 & $\mathrm{~F}$ & 25 & NA & NA & 25 & 25 & $\begin{array}{l}\text { Chronic } \\
\text { severe }\end{array}$ & $\begin{array}{l}\mathrm{L} \text { foot, } \mathrm{L} \\
\text { wrist and } \\
\mathrm{R} \text { hand }\end{array}$ & Infliximab \\
\hline Our case & Heraklion & Greece & 2020 & M & 43 & 19 & 27 & 25 & 46 & $\begin{array}{l}\text { Chronic } \\
\text { severe }\end{array}$ & $\mathrm{R}$ lower leg & Infliximab \\
\hline
\end{tabular}

F, female; M, male; PG, pyoderma gangrenosum; IBD, inflammatory bowel disease; UC, ulcerative colitis; GCAP, granulocyte apheresis; td: three times a day

Together with erythema nodosum, PG represents the most common dermatologic disorder accompanying IBD, which comprises UC and Crohn's disease [1]. PG has been reported to occur in $2-12 \%$ of IBD patients and may either precede colitis or occur at any stage of the disease, even after the colon has been removed $[7,8]$.

In most patients, symptoms of UC precede PG, and bowel disease relapses frequently correlate with worsening of the skin lesions. However, PG is not closely related to the activity of UC and may persist for long periods while bowel disease is quiescent [1]. PG is also associated with Crohn's disease, but the prevalence of this association is lower than that observed for UC [7].

Here we have presented a rare case of PG developing in a 43-year-old male patient with a past medical history of UC and chronic refractory pouchitis, 21 years after surgery with IPAA, who responded well to treatment with IFX. To the best of our knowledge, there are only 4 other cases of pouchitis-related PG after IPAA in UC (Table 1) [3-6].

Pouchitis is the most frequently observed long-term complication of IPAA [2]. Among patients who have undergone IPAA, the reported incidence of pouchitis ranges from $23-59 \%[2,7]$. The pathogenesis of pouchitis is unclear, but it is hypothesized to result from an abnormal immune response to altered luminal and/or mucosal bacteria in genetically susceptible hosts [2]. Smoking is associated with an increased risk of acute pouchitis but a decreased risk of chronic pouchitis. Sometimes it is difficult to distinguish between chronic refractory pouchitis and Crohn's disease of the pouch, but in our case no findings of Crohn's disease were noticed during the long-term follow up.

Our case highlights that infliximab might be an effective and well-tolerated treatment for pouchitis-associated PG. Clinicians should be aware of the risk of PG in patients suffering from pouchitis and developing rapidly extensive painful ulcers. Furthermore, the therapeutic choice should take into consideration the effectiveness of IFX on the inflammatory background, which sustains both intestinal and skin disease in these types of patients.

\section{References}

1. Ruocco E, Sangiuliano S, Gravina AG, Miranda A, Nicoletti G. Pyoderma gangrenosum: an updated review. J Eur Acad Dermatol Venereol 2009;23:1008-1017.

2. Lohmuller JL, Pemberton JH, Dozois RR, Ilstrup D, van Heerden J. Pouchitis and extraintestinal manifestations of inflammatory bowel disease after ileal pouch-anal anastomosis. Ann Surg 1990;211:622-627.

3. Abdelrazeq AS, Lund JN, Leveson SH. Pouchitis-associated pyoderma gangrenosum following restorative proctocolectomy for ulcerative colitis. Eur J Gastroenterol Hepatol 2004;16:1057-1058.

4. Yanaru-Fujisawa R, Matsumoto T, Nakamura S, et al. Granulocyte apheresis for pouchitis with arthritis and pyoderma gangrenosum after restorative proctocolectomy for ulcerative colitis: a case report. Inflamm Bowel Dis 2005;11:780-781.

5. Molnar T, Farkas K, Nagy F, Wittmann T. Successful use of infliximab for treating fistulizing pouchitis with severe extraintestinal manifestation: a case report. Inflamm Bowel Dis 2008;14:1752-1753.

6. Satake M, Sakuraba H, Hiraga H, et al. Successful treatment with tacrolimus of refractory pyoderma gangrenosum with pouchitis after restorative proctocolectomy for ulcerative colitis. Immunol Med 2018;41:142-146.

7. Goldstein NS, Sanford WW, Bodzin JH. Crohn's-like complications in patients with ulcerative colitis after total proctocolectomy and ileal pouch-anal anastomosis. Am J Surg Pathol 1997;21:1343-1353.

8. Holmlund DE, Wählby L. Pyoderma gangrenosum after colectomy for inflammatory bowel disease. Case report. Acta Chir Scand 1987;153:73-74. 